\title{
Metal-Free Aryl Cross-Coupling Directed by Traceless Linkers
}

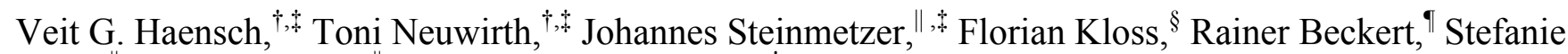 \\ Gräfe, Stephan Kupfer, ${ }^{\|, *}$ and Christian Hertweck ${ }^{\dagger} *$ \\ ${ }^{\dagger}$ Department of Biomolecular Chemistry, Leibniz Institute for Natural Product Research and Infection Biology, HKI, Beu- \\ tenbergstr. 11a, 07745 Jena, Germany \\ "Institute for Physical Chemistry, Friedrich Schiller University Jena, Helmholtzweg 4, 07743 Jena, Germany \\ ${ }^{\S}$ Transfer Group Antiinfectives, Leibniz Institute for Natural Product Research and Infection Biology (HKI), 07745 Jena, \\ Germany. \\ "Institute for Organic and Macromolecular Chemistry (IOMC), Friedrich Schiller University Jena, 07743 Jena, Germany \\ KEYWORDS Biaryls; Cross-coupling; Photochemistry; Sulfonamides; Synthetic methods; Photosplicing; TDDFT
}

\begin{abstract}
The metal-free, highly selective synthesis of biaryls poses a major challenge in organic synthesis. We report the scope and mechanism of a promising new approach to (hetero)biaryls by the photochemical fusion of aryl substituents tethered to a traceless linker (photosplicing). Interrogating photosplicing with varying reaction conditions and comparison of diverse synthetic probes (40 examples, including a suite of heterocycles) showed that the reaction has a surprisingly broad scope and involves neither metals nor radicals. Quantum chemical calculations revealed that the $\mathrm{C}-\mathrm{C}$ bond is formed by an intramolecular photochemical process that involves an excited singlet state and the traverse of a five-membered transition state, thus warranting consistent $i p$ so-ipso-coupling fidelity. These results demonstrate that photosplicing is a unique aryl cross-coupling method in the excited state that can be applied to synthesize a broad range of biaryls.
\end{abstract}

The enormous economic role of biaryls in chemical and pharmaceutical products ${ }^{1}$ has propelled the development of a large number of cross-coupling methodologies. ${ }^{2}$ In general, metal catalysis offers great fidelity in terms of substrate tolerance and scalability of biaryl synthesis. ${ }^{3}$ Yet, in large-scale manufacture and the production of bioactive ingredients, removal of heavy metal impurities, catalyst recycling, and the provision of suitable starting materials can be challenging and costly. ${ }^{4,5}$ Thus, there is a high demand for alternative, metalfree cross-coupling methods. ${ }^{6-10}$ Most known metal-free approaches, however, suffer from narrow scope or low selectivity. Recently, we discovered that bis-aryl-substituted sulfonamides can be contracted photochemically, thereby permitting the regioselective fusion of two aryl groups. ${ }^{11}$ Using a UV photoreactor, we demonstrated the preparative applicability of the reaction in the synthesis of building blocks for a series of clinically relevant pharmaceuticals. Remarkably, all required anchor groups to tether the aryls as sulfonamides are cleanly extruded as volatile fragments (ammonia, sulfur dioxide, formaldehyde), yielding the ipso-ipso-coupled biaryl product (Figure 1A). Still, the scope and the molecular mechanism of this unusual photosplicing reaction have remained elusive. Here we address these important questions by a combination of chemical synthesis and quantum mechanical considerations, and present photosplicing as a highly versatile method for the selective, metal-free preparation of biaryls.

To test the scope of the photochemical aryl coupling, we prepared and compared a library of 30 sulfonamides with different substitution patterns and chemically diverse substituents at both aryl residues (Figure 1B). Upon $254 \mathrm{~nm}$ irradia- tion of these compounds we found that $p$-alkyl carboxylatesubstituted educts give best results, in particular when this substituent is located at the benzylic residue (blue). In lieu of a carboxyester, various electron-withdrawing groups (nitrile, trifluoromethyl, acyl, and formyl) can be employed. Likewise, electron-releasing substituents (methoxy, benzyloxy, dimethylamino, methyl) afford the desired products, albeit in reduced yields. As to the benzenesulfonyl residue (green), photosplicing tolerates methyl, ethyl, methoxy, benzyloxy, hydroxyl, fluoro, chloro, nitrile, carboxymethyl, acetyl, dimethylamino, methyl thioether, and trifluoromethyl moieties. It should be highlighted that photosplicing is even possible in the presence of two ortho-methoxy or benzyloxy groups $(\mathbf{2 g}, \mathbf{2 j})$. In contrast, two ortho-methyl groups (2ad) and the lack of substituents (2p) hamper the reaction and yield only traces of the corresponding biaryls. Overall, photosplicing proved to be highly versatile as it tolerates a broad range of photostable substituents. Surprisingly, even substrates with reversed polarity, such as $1 \mathbf{i}$ and 1aa, afford the corresponding biaryls.

To evaluate the possibility of coupling heteroaromatic units we prepared a suite of sulfonamides with pyridine, imidazole, thiophene and its benzannulated derivatives (Figure 1C). Irradiation with UV light (254 $\mathrm{nm}$ ) led to the expected photosplicing products in moderate to excellent yields (Figure 1D). Best results were obtained with pyridine and imidazole moieties. These findings illustrate the remarkably broad substrate scope for photosplicing. 
A

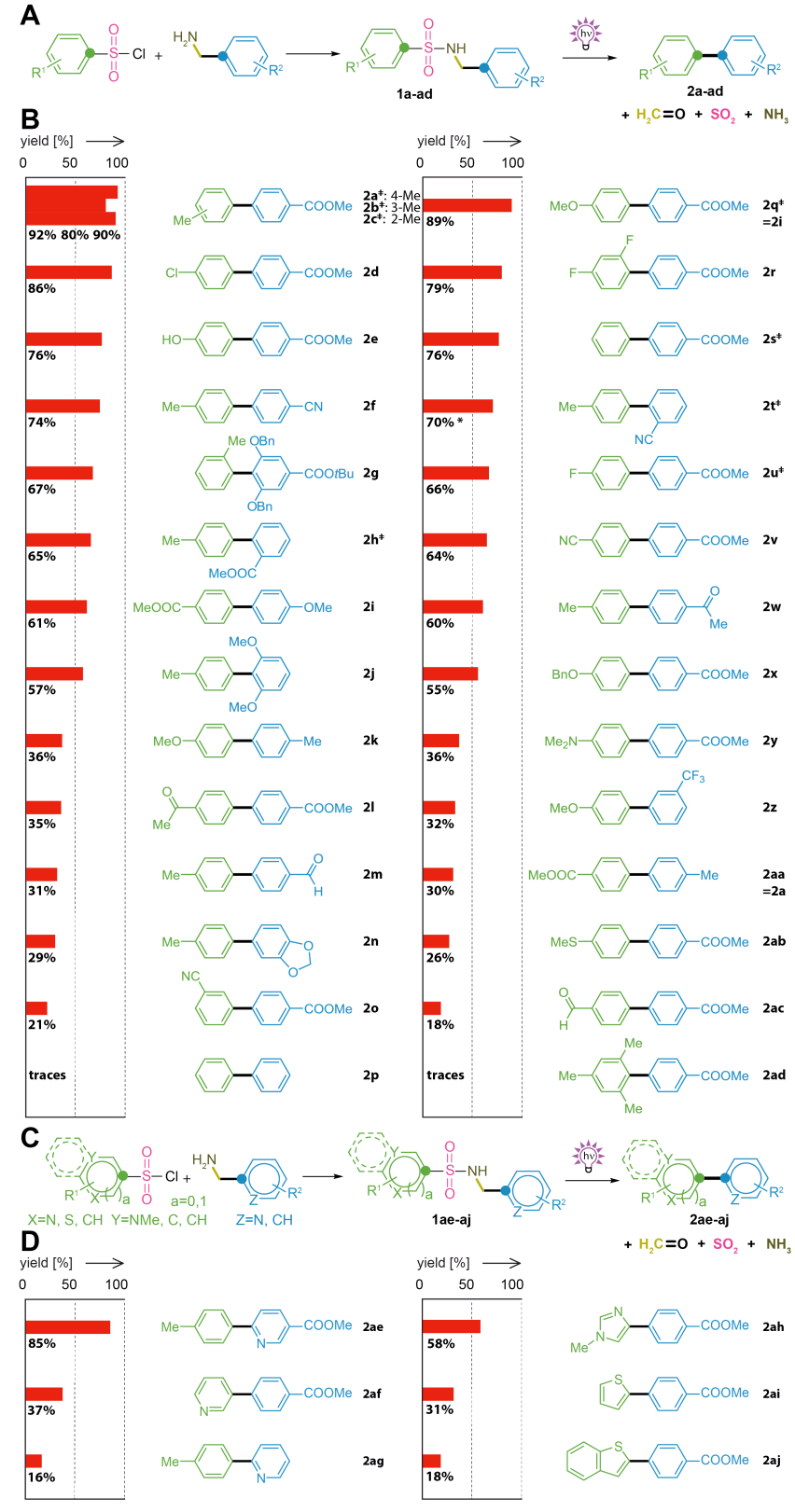

Figure 1. General scheme and scope of photosplicing. A) General reaction scheme. B) Biaryl target compounds and comparison of yields obtained by photosplicing. C) General reaction scheme for heterobiaryls. D) Heterobiaryl target compounds and comparison of yields obtained by photosplicing. *Based on recovered starting material. ${ }^{\dagger}$ Known example, included for comparison.

For the photoactivated aryl coupling, various reaction mechanisms are conceivable. UV light excitation could potentially lead to the homolytic fission of the linker followed by formation of carbon-centered radicals and coupling of phenyl radicals (Figure 2A, I), ${ }^{12}$ or the observed ipso-ipso substitution could involve intermolecular reactions favored by dispersive interactions (Figure 2A, II). ${ }^{13}$ Although no metal additives were used, trace metal impurities in solvents and reagents could be sufficient to facilitate photocatalytic effects (Figure 2A, III). ${ }^{14}$ Alternatively, the biaryl could be formed in analogy to a radical Smiles type rearrangement (Figure 2A, IV) ${ }^{15,16}$ Another plausible route could involve an intramolecular ipso-ipso attack of the excited sulfonamide leading to a five-membered transition state or intermediate, which undergoes further fragmentation (Figure 2A, V).

To unravel whether UV-irradiation of sulfonamide 1a afforded reactive aryl radicals in a Norrish type I reaction, ${ }^{17}$ first we meticulously analyzed the reaction mixture; yet, biaryls that would arise from random radical recombination processes could not be detected. Furthermore, neither radical starters such as dibenzoyl peroxide (DBPO) or azobisisobutyronitrile (AIBN), nor the mixture of tributyltinhydride and AIBN, which is used for the radical Smiles rearrangement, ${ }^{18}$ initiated the biaryl coupling (Figure 2B). Moreover, we found that the course of the photoreaction is not affected in the presence of the radical quencher 2,6-di-tert-butyl-4-methylphenol (BHT), ${ }^{19}$ even when added in large excess (Figure 2C). To trap potential intermediary radicals, excess of toluene to the photoreaction with sulfonamide 1s was added. Even when using toluene as co-solvent in acetonitrile $(v / v=50: 50)$, no side products of the coupling reaction were detectable (Figure 2D). Finally, we installed a cyclopropyl, which serves as a radical trap, ${ }^{20}$ at the linker and noted that this unit does not hamper the photoreaction (Figure 2G). Thus, the involvement of (longliving) radicals in the course of the aryl coupling can be excluded.

To investigate the potential involvement of metal catalysts in the aryl coupling, we added different late d-block metals (Fe, Co, Ni, Pd, Pt, Ir, Ru, Cu, Rh, Os) that are used for cross couplings $^{21,22}$ to sulfonamide 1a. In the absence of light, biphenyls were not formed, and after irradiation no increased ratio of sulfonamide to biphenyl could be observed (Figure $2 \mathrm{E}$ ). These findings provide strong support for a metal-free reaction mechanism.

As an alternative to the templating effect of metals, we reasoned that the aromatic rings of the sulfonamide educts could be positioned by dispersive interactions. To address the possibility of potential intermolecular reactions, we prepared a pair of sulfonamides with complementary substitution and monitored the course of the photoreaction using a mixture of educts. The photoreaction of sulfonamide 1a and 1ak in one batch with the same molar ratio showed no photoproducts with mixed substituents (Figure 2F). This cross-reactivity experiment is a clear indication for an intramolecular recombination. Consequently, the linker plays a key role in the aryl coupling.

To estimate the impact of $C$ - and $N$-substitutions in the linker and thus the facilitation of the ipso-carbon attack by tautomer formation, we exchanged all protons by methyl groups (Figure 2G). Therefore, we prepared an $N$-methyl sulfonamide (1al), as well as sulfonamides with two methyl groups at the carbon of the linker (1am). As both substrates afforded the corresponding biphenyl (2a), we concluded that the influence of potential tautomers on the reaction is negligible. 
A
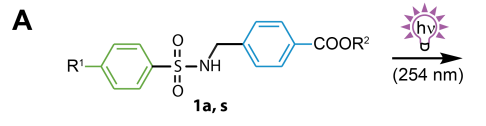

$\mathrm{R}^{1}-\mathrm{COOR}^{1}$ $\begin{array}{ll}2 a, s & \text { a: } R^{\prime}=R^{2}=M e \\ \text { s: } R^{\prime}=H, R^{2}=M e\end{array}$
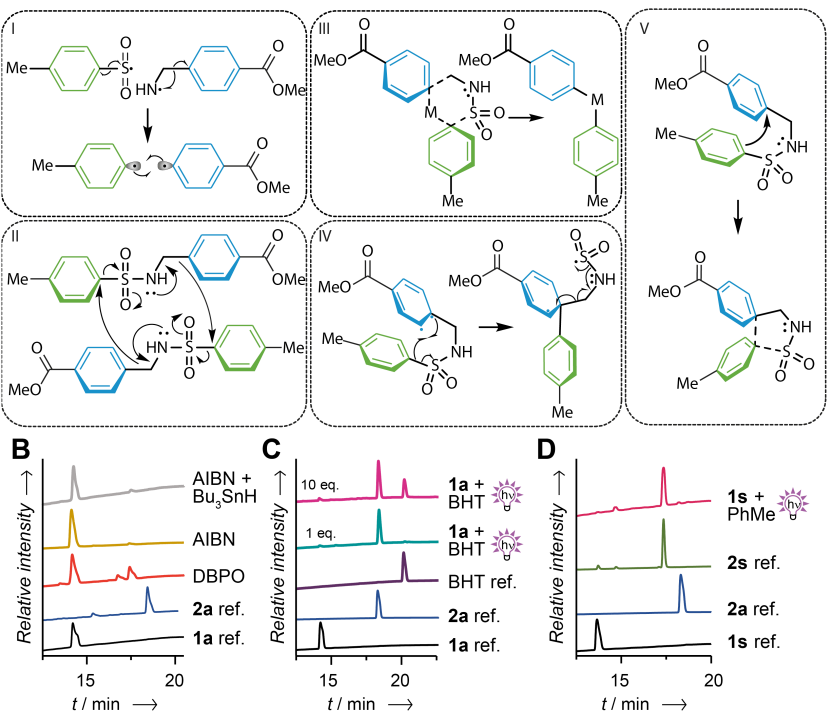

$c$

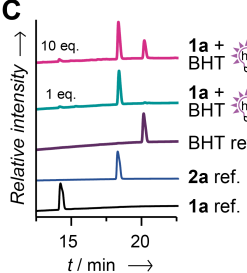

D

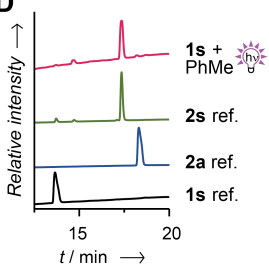

$E_{0}$

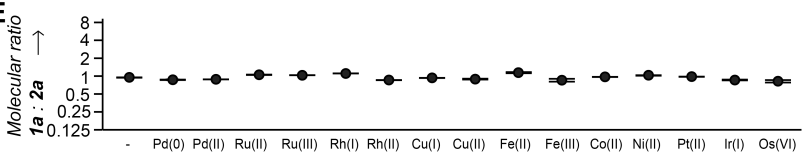

$\mathbf{F}$

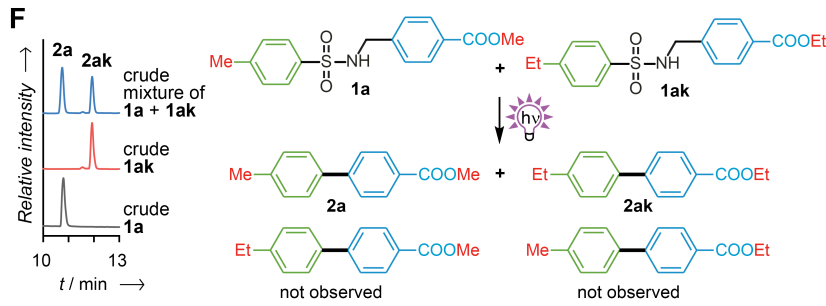

$\mathbf{G}$

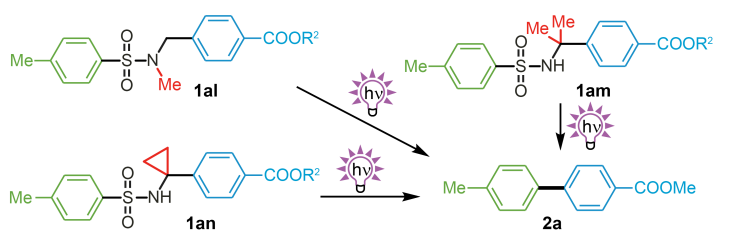

Figure 2. Evaluation of potential reaction mechanisms. A) General reaction scheme and different mechanistic scenarios for the photosplicing process I) radical, II) intermolecular, III) metalcatalyzed, IV) Smiles-like rearrangement, V) regiochemically controlled intramolecular reaction; B) impact of radical initiators, C) radical quenchers, D) reactive solvent, E) and addition of metals, F) cross-reactivity, G) modification of linkers to hamper tautomer formation and to install a radical trap.

To elucidate the mechanism underlying the photosplicing at a molecular level, we performed quantum chemical simulations, density functional theory (DFT) and its time-dependent extension (TD-DFT). As models we investigated the lightdriven formation of biphenyl 2a from sulfonamides 1a and 1aa, respectively, featuring inverse electronic structures. Initially, we calculated the potential energies of the educt states (1a and 1aa) by GFN-xTB, ${ }^{23}$ which permits a detailed analysis of the possible conformers in the ground state. The energies of selected conformers were verified by coupled cluster calculations, ${ }^{24}$ and were subsequently analyzed by means of a principal component analysis (Figure S9-S12).
Relaxed scans along the dihedral angle $\varphi$ (given by C2-N3-S4-C5) revealed that the flexible sulfonamide linker adapts preferably linear and U-shaped horseshoe conformers (Figure 3A). The relaxed potential energy curves (PECs) obtained by DFT (Figure 3A) indicated that in 1a and 1aa the horseshoe conformer $\left(\varphi=80^{\circ}, \varphi=70^{\circ}\right.$ for 1aa) is energetically more favored (by $10 \mathrm{~kJ} / \mathrm{mol}$ ) than the linear conformer $\left(\varphi=-70^{\circ}\right)$. The 1a horseshoe conformer exhibits a short $\mathrm{C} 1-$ $\mathrm{C} 5$ distance $(326 \mathrm{pm})$, which is pivotal for the $\mathrm{C}-\mathrm{C}$ coupling of the aromatic rings.

To evaluate the potential course of the aryl coupling, we subsequently assessed the formation of photoproducts $\mathbf{2 a}$ and $2 \mathbf{a a}$ along a reaction pathway given by an intrinsic reaction coordinate (IRC). The IRC connects the educt and product states via a cyclic, C1-C2-N3-S4-C5-containing transition state (TS) optimized by DFT. Within the five-membered TS of the reaction from $\mathbf{1 a}$ to $\mathbf{2 a}$ the $\mathrm{C} 1-\mathrm{C} 5$ distance decreases to $164 \mathrm{pm}$, which is associated to $\mathrm{C}-\mathrm{C}$-bond formation. Simultaneously, partial cleavage of the $\mathrm{C}-\mathrm{S}$ bond is indicated by the increasing bond length from 176 to $239 \mathrm{pm}$ (Figure 3B). In stark contrast, changes within the linker are less pronounced until the TS is reached. Only upon relaxation towards the product state, and thus formation of the photoproduct (2a), the $\mathrm{S}-\mathrm{N}$ and $\mathrm{C} 1-\mathrm{C} 2$ distances are increased, indicating the extrusion of $\mathrm{SO}_{2}$ and $\mathrm{CH}_{2} \mathrm{NH}$, which can hydrolyze into ammonia and formaldehyde (Figure 3B). According to our calculations, the formation of the TS requires an activation energy of $4.08 \mathrm{eV}$ in the ground state.

To gain insight into the excited states involved in the photoreaction, we first studied the low-lying excited singlet states of 1a and 1aa within the Franck-Condon region. The simulated absorption spectrum of 1a (Figure 3C) reveals several bright states, i.e. $\mathrm{S}_{2}$ at $5.30 \mathrm{eV}(234 \mathrm{~nm})$ and $\mathrm{S}_{3}$ at $5.40 \mathrm{eV}$ $(230 \mathrm{~nm})$, in the vicinity of the irradiating light source centered at $4.88 \mathrm{eV}(254 \mathrm{~nm})$. Light-driven coupling of the aryl moieties is feasible by the leading transition underlying $\mathrm{S}_{2}$ of 1a, which features a bonding character between the carbon atoms $\mathrm{C} 1$ and $\mathrm{C} 5$ (Figure $3 \mathrm{C}$ ). In addition, the $\mathrm{S}_{2}$ state coincides with the experimentally determined maximum turnover at $5.28 \mathrm{eV}(235 \mathrm{~nm}) .{ }^{11}$ Evolution of the adiabatic PECs of the low-lying excited states reduces the energetic barrier to enable photosplicing of 1a within the excited states to only $0.69 \mathrm{eV}$ along the IRC. It is very remarkable that reversal of the substrate polarity (1aa in lieu of $\mathbf{1 a}$ ) leads to a congruent model and activation barrier $\left(0.77 \mathrm{eV}\right.$ for $\left.\mathrm{S}_{1}\right)$.

To corroborate the quantum chemical simulations, we chose 1p as reference, since the bis-phenyl-substituted sulfonamide yields merely traces of the photoproduct (2p). Compared to $\mathbf{1 a}$ and 1aa, we found that in $\mathbf{1 p}$ the determined excited states are shifted hypsochromically and with lowered oscillator strengths (Table S3). Therefore, the disadvantageous excited state properties of $\mathbf{1 p}$ prevent an efficient photoreaction. The computational results are in full accordance with the experimental observations and thus allowed to elucidate the mechanism of photosplicing.

Taken together, our quantum chemical simulations show that electron-withdrawing and electron-donating groups are a prerequisite of the photoreaction because these substituents control the energy of the excited states and determine the overlap with the excitation wavelength. It is remarkable that the substitution pattern and thus polarity of the sulfonamide 
neither have an impact on the IRCs nor on energies of the excited state relaxation cascades.

The course of the photoreaction can be illustrated by means of the frontier orbitals of 1a (Figure S13) contributing to $S_{2}$. Specifically, the highest occupied molecular orbital (HOMO) exhibits antibonding character between $\mathrm{C} 1$ and $\mathrm{C} 5$ whereas the lowest unoccupied molecular orbital (LUMO) shows bonding character between these two carbon atoms. The photo-induced population of the LUMO lowers the activation energy substantially and enables the formation of 2a. The small energy gap $(1.0 \mathrm{eV})$ between the ground and the excited state $\left(\mathrm{S}_{1}\right)$ in the vicinity of the TS facilitates the relaxation into the product state.

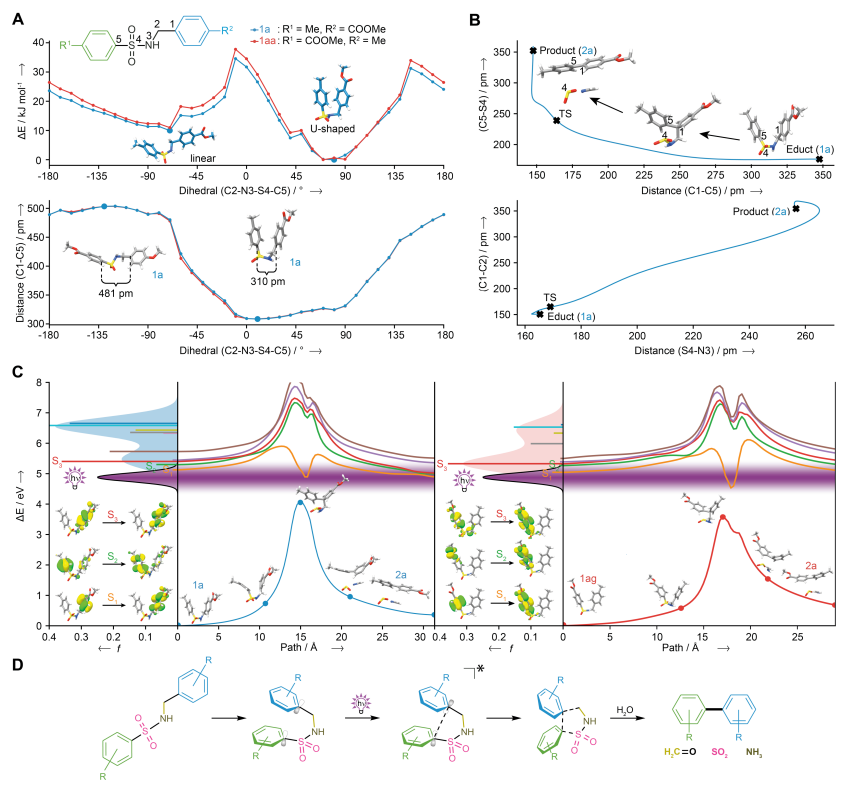

\section{—double spaced figure, see original below-}

Figure 3. In silico analysis of photosplicing. A) Relaxed scan along the dihedral angle of the linker from sulfonamide 1a and 1aa with energy differences and $\mathrm{C} 1-\mathrm{C} 5$ distance, B) distance of C1-C5 related to $\mathrm{C} 5-\mathrm{S} 4$ and distance of $\mathrm{S} 4-\mathrm{N} 3$ related to $\mathrm{C} 1-\mathrm{C} 2$ during the photosplicing reaction, $\mathrm{C}$ ) reaction coordinate in the ground state and low-lying excited (singlet) states of the photosplicing reaction with the calculated absorption spectra and the emission of the light source, D) deduced model for the reaction mechanism of photosplicing.

In conclusion, we have demonstrated that photosplicing is a highly versatile method for the selective synthesis of a broad range of biaryls. With 40 examples, we show that a large number of residues with different regiochemical orientation and even heteroaromatic rings are tolerated. Notably, photosplicing is an exceptional traceless ipso-ipso-selective aryl cross-coupling method, which is truly metal-free and, more importantly, independent of free radical formation. By combining synthesis and computational methods we have shed light on the mechanism of this novel photochemical reaction. It is initiated by the formation of a U-shaped conformation that leads to a cyclic intermediary state. With only few exceptions, the energy of UV-C light source is sufficiently high to achieve the population of excited states, which drive the reaction towards the targeted biaryls. Beyond providing a general model for photosplicing, our results demonstrate that this highly selective and versatile sulfonamide contraction is an important addition to the current toolbox for metal-free biaryl syntheses.

\section{ASSOCIATED CONTENT}

\section{Supporting Information}

Full methods, detailed synthetic procedures and physicochemical characterization of new compounds including NMR spectra and quantum chemical simulations are available in the Supporting Information. The Supporting Information is available free of charge on the ACS Publications website.

\section{AUTHOR INFORMATION}

\section{Corresponding Author}

* E-mail: stephan.kupfer@uni-jena.de (calculations); christian.hertweck@hki-jena.de (synthesis)

\section{Author Contributions}

C.H., F.K., R.B. and S.G. designed research. V.G.H., and T.N. performed synthetic experiments and analyses. J.S. and S.K. performed calculations and generated models. The manuscript was written through contributions of all authors; C.H. wrote the final version. All authors have given approval to the final version of the manuscript.

\$These authors contributed equally.

\section{Funding Sources}

We thank the Deutsche Forschungsgemeinschaft (Leibniz Prize, for C.H.) and the Federal Ministry of Education and Research (BMBF) of Germany within the program InfectControl 2020 (FKZ 03ZZ0803 for F.K.) for financial support.

\section{ACKNOWLEDGMENT}

We thank A. Perner for MS analyses and H. Heinecke for NMR measurements.

\section{REFERENCES}

(1) Horton, D. A.; Bourne, G. T.; Smythe, M. L. Chem. Rev. 2003, 103, 893 .

(2) Johansson Seechurn, C. C. C.; Kitching, M. O.; Colacot, T. J.; Snieckus, V. Angew. Chem. Int. Ed. 2012, 51, 5062.

(3) Biffis, A.; Centomo, P.; Del Zotto, A.; Zecca, M. Chem. Rev. 2018, 118, 2249.

(4) Garrett, C. E.; Prasad, K. Adv. Synth. Catal. 2004, 346, 889.

(5) O'Brien, H. M.; Manzotti, M.; Abrams, R. D.; Elorriaga, D.; Sparkes, H. A.; Davis, S. A.; Bedford, R. B. J. N. C. Nat. Catal. 2018, $1,429$.

(6) Hilton, M. C.; Zhang, X.; Boyle, B. T.; Alegre-Requena, J. V.; Paton, R. S.; McNally, A. Science 2018, 362, 799.

(7) Sun, C.-L.; Li, H.; Yu, D.-G.; Yu, M.; Zhou, X.; Lu, X.-Y.; Huang, K.; Zheng, S.-F.; Li, B.-J.; Shi, Z.-J. Nat. Chem. 2010, 2, 1044.

(8) Sun, C.-L.; Shi, Z.-J. Chem. Rev. 2014, 114, 9219.

(9) Piazzolla, F.; Colognese, F.; Temperini, A. Curr. Org. Chem. 2018, 22, 2537.

(10) De Carolis, M.; Protti, S.; Fagnoni, M.; Albini, A. Angew. Chem., Int. Ed. 2005, 44, 1232.

(11) Kloss, F.; Neuwirth, T.; Haensch, V. G.; Hertweck, C. Angew. Chem. Int. Ed. 2018, 57, 14476.

(12) Weiss, B.; Dürr, H.; Haas, H. J. Angew. Chem. Int. Ed. 1980, 19, 648 .

(13) Martinez, C. R.; Iverson, B. L. Chem. Sci. 2012, 3, 2191.

(14) Leadbeater, N. E. Nat. Chem. 2010, 2, 1007.

(15) Snape, T. J. Chem. Soc. Rev. 2008, 37, 2452. 
(16) Holden, C. M.; Sohel, S. M.; Greaney, M. F. Angew. Chem. Int. Ed. 2016, 55, 2450.

(17) Scaiano, J. C.; Stamplecoskie, K. G.; Hallett-Tapley, G. L. J. C. C. Chem. Comm. 2012, 48, 4798.

(18) Allart-Simon, I.; Gérard, S.; Sapi, J. Molecules 2016, 21, 878

(19) Yehye, W. A.; Rahman, N. A.; Ariffin, A.; Hamid, S. B. A.; Alhadi, A. A.; Kadir, F. A.; Yaeghoobi, M. Eur. J. Med. Chem. 2015, 101, 295.

(20) Paul, B.; Das, D.; Ellington, B.; Marsh, E. N. G. J. Am Chem. Soc. 2013, 135, 5234.

(21) Liu, C.; Zhang, H.; Shi, W.; Lei, A. Chem. Rev. 2011, 111, 1780 .

(22) Ackermann, L.; Vicente, R.; Kapdi, A. R. Angew. Chem. Int. Ed. 2009, 48, 9792.

(23) Grimme, S.; Bannwarth, C.; Shushkov, P. J. Chem. Theory Comput. 2017, 13, 1989.

(24) Riplinger, C.; Neese, F. J. Chem. Phys. 2013, 138, 034106. 


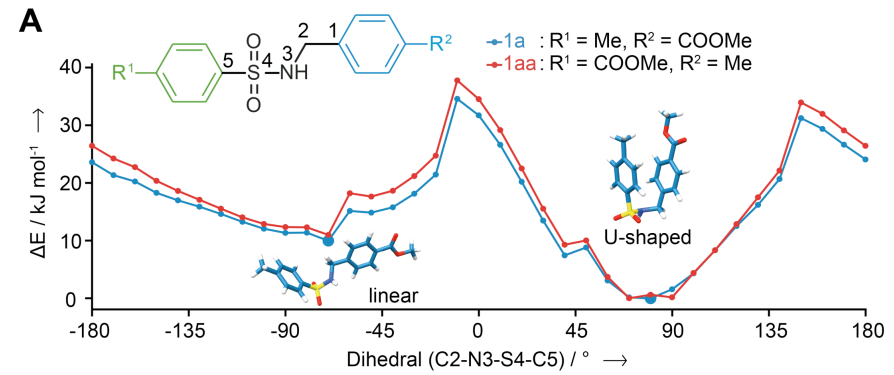

B
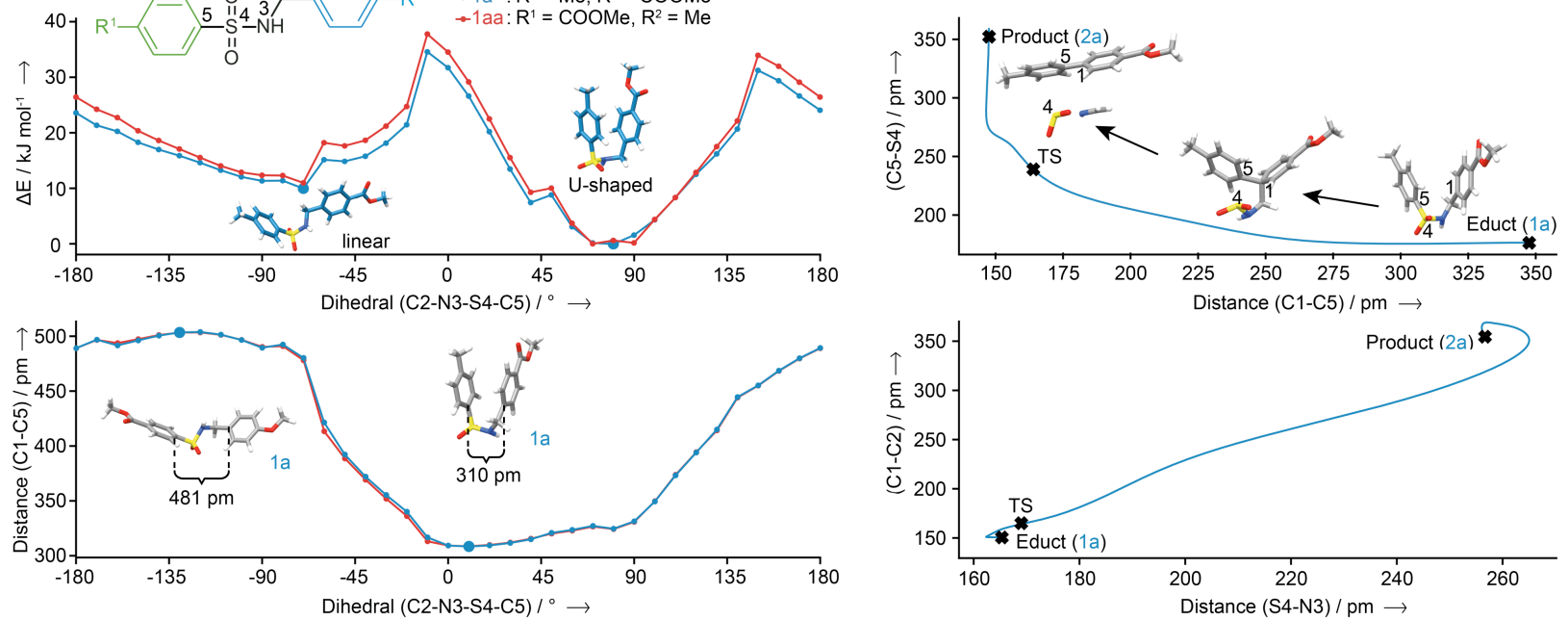

C

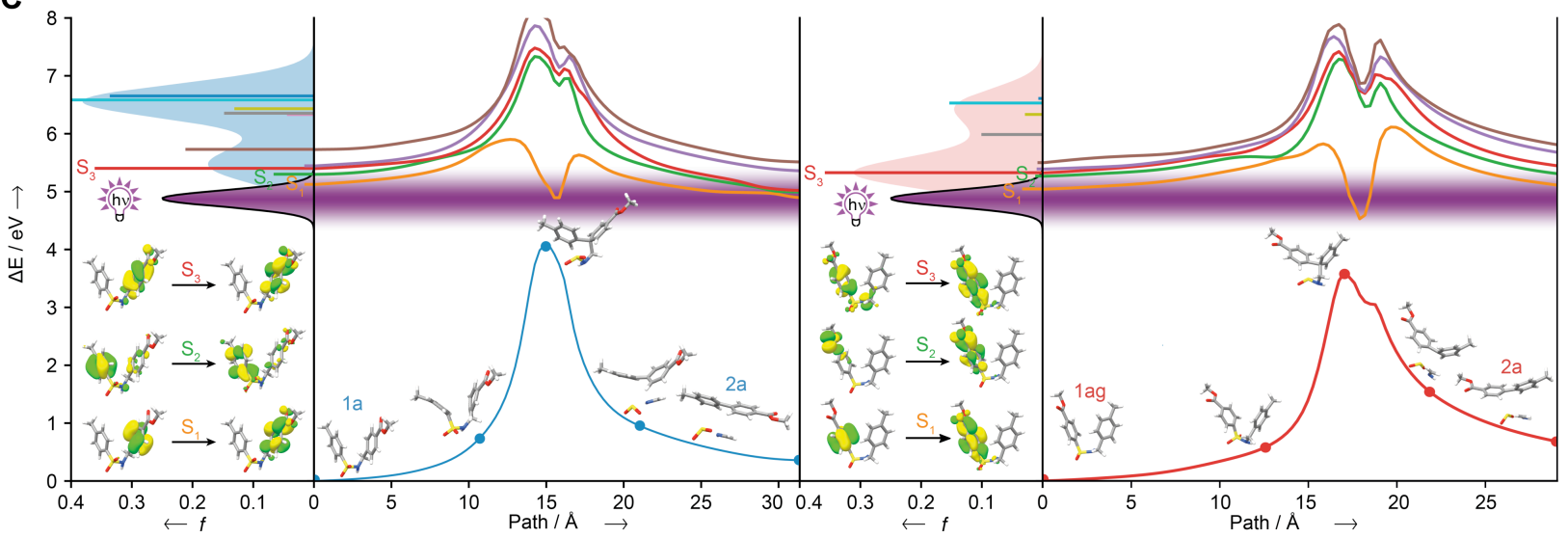

D

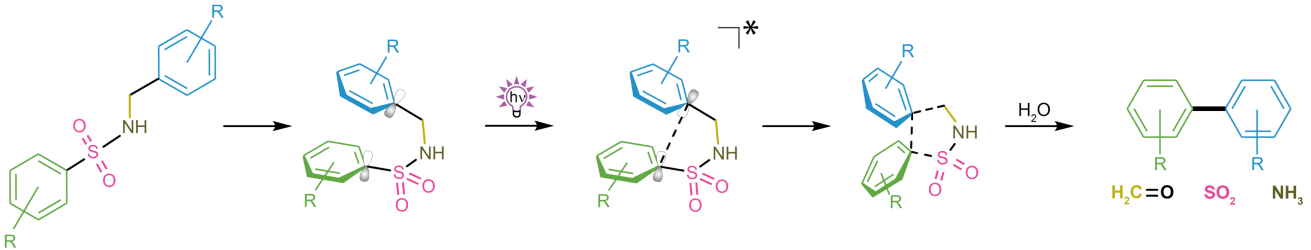

Figure 3. In silico analysis of photosplicing. A) Relaxed scan along the dihedral angle of the linker from sulfonamide 1a and 1aa with energy differences and $\mathrm{C} 1-\mathrm{C} 5$ distance, B) distance of C1-C5 related to C5-S4 and distance of S4-N3 related to C1-C2 during the photosplicing reaction, $\mathrm{C}$ ) reaction coordinate in the ground state and low-lying excited (singlet) states of the photosplicing reaction with the calculated absorption spectra and the emission of the light source, D) deduced model for the reaction mechanism of photosplicing. 